\title{
La Comtesse de Ségur ou l'universel du dialogue
}

\section{Catherine Boré}

\section{(2) OpenEdition}

\section{Journals}

Édition électronique

URL : http://journals.openedition.org/genesis/3995

DOI : 10.4000/genesis.3995

ISSN : 2268-1590

\section{Éditeur :}

Presses universitaires de Paris Sorbonne (PUPS), Société internationale de génétique artistique littéraire et scientifique (SIGALES)

\section{Édition imprimée}

Date de publication : 1 juin 2019

Pagination : 37-54

ISSN : 1167-5101

\section{Référence électronique}

Catherine Boré, «La Comtesse de Ségur ou l'universel du dialogue », Genesis [En ligne], 48 | 2019, mis en ligne le 01 juin 2020, consulté le 25 janvier 2021. URL : http://journals.openedition.org/genesis/ 3995 ; DOI : https://doi.org/10.4000/genesis.3995 


\title{
La Comtesse de Ségur ou l'universel du dialogue
}

\author{
Catherine Boré
}

$\mathrm{D}$ ans cet article, nous avons choisi pour fil conducteur le dialogue, compris non seulement comme forme générique ou linguistique, mais comme un invariant anthropologique permettant une clé de lecture de l'invention dans l'élaboration scripturale. Nous associons à cet invariant l'un des écrivains les plus emblématiques de la littérature de jeunesse, Sophie de Ségur, née Rostopchine, la Comtesse de Ségur (désormais abrégée en Ségur) dont nous commenterons cet aspect de l'œuvre, à partir du manuscrit des Malheurs de Sophie.

Il s'agit des débuts de Ségur romancière de fiction, qui, après Nouveaux Contes de fées, avait fait paraître en 1858 le premier volume de la trilogie de Fleurville, Les Petites Filles Modèles. La rédaction des Malheurs de Sophie commence vraisemblablement en février-mars 18581, interférant avec le manuscrit des Vacances écrit simultanément. Dans cette trilogie, prévaut déjà le dialogue théâtralisé 2 , forme choisie dès le premier roman, et particulièrement affirmée dans Les Malheurs de Sophie, où elle prend d'autant plus de force que les chapitres sont courts et destinés à de plus jeunes enfants.

Pourquoi le dialogue? Et en quoi cette forme serait-elle emblématique de l'écriture de la littérature d'enfance, qui est aussi, dans le cas de Ségur, enfance de l'écriture?

Pour répondre à ces questions, nous nous plaçons naturellement d'abord sous le patronage de Bakhtine, en associant dialogue et dialogisme car, bien que distincts, les deux termes sont liés par une représentation commune du langage, considéré comme aimanté par les deux pôles de la question et de la réponse. Dans le dialogue en face à face, particulièrement illustré chez Ségur, sont représentés ou se présentent des locuteurs/allocutaires différents qui manifestent leur altérité par le fait même d'énoncer et de répondre. Le fil narratif est ainsi émaillé de discours de personnages différents, eux-mêmes traversés de voix autres. Et même lorsque le locuteur n'a pas d'interlocuteur désigné et se parle à lui-même, qu'il reprend et soupèse ses propres mots, s'exerce un «autodialogisme»; nous le verrons plus loin avec les courts monologues de Sophie.

Mais cet article s'inscrit avant tout dans la perspective d'une écriture de l'enfance, comprise tout autant comme une écriture destinée à l'enfance que comme une écriture illustrant certaines de ses particularités. Parmi celles-ci, nous avons retenu l'élaboration de la pensée enfantine. C'est pourquoi nous nous référerons principalement aux analyses du psychologue russe Vygotski concernant la genèse de la pensée chez l'enfant.

1. Rémi Saudray et François Labadens, «Et pourquoi pas une trilogie?» Cahiers Séguriens, n 9 , Association des Amis de la Comtesse de Ségur, 2010, p. 15.

2. Nous dénommerons ainsi désormais la présentation des échanges dialogués entre personnages sous la forme de répliques surmontées du nom du locuteur, comme le sont les personnages au théâtre, et non sous la forme, habituelle dans les romans, de dialogues introduits par un narrateur désignant le locuteur. 
Les thèses de Vygotski sur le langage et la pensée partent précisément du dialogue et de l'interaction de l'enfant avec l'adulte comme d'une condition de sa capacité à élaborer une pensée propre. C'est dans la distance entre la pensée d'autrui et la réponse ou compréhension qu'en retient l'enfant que peut s'effectuer un mouvement de pensée propre.

Au moment où l'enfant commence à penser, il passe par une phase du langage qui a été nommée «égocentrique». Au contraire de Piaget, Vygotski considère ce langage comme la manifestation externe d'un effort pour penser : par la suite, en effet, l'enfant l'abandonnera pour intérioriser ce langage et le confronter aux représentations préconstruites dont il dispose. Ce langage intérieur contient des éléments d'élaboration de résolution de problèmes et se présente, si l'on en croit les expériences menées par Vygotski, comme intégrant les questions et les réponses que se fait à soi-même le locuteur, de façon synthétique et compréhensible par lui seul. Vygotski a également rapproché «brouillon mental» ou «langage intérieur» (Bakhtine/Volochinov parlera de «discours intérieur») et brouillon écrit. Vygotski pense ce brouillon mental, ce dialogue interne, comme un instrument sémiotique analogue au dialogue, doté d'une fonction sociale et transformatrice de conduites ${ }^{3}$.

Le dialogue, dans ce sens particulier, est donc premier. Il imprègne profondément la psyché enfantine. C'est pourquoi les discussions externes entre personnages, et internes aux personnages eux-mêmes, sont particulièrement intéressantes dans la littérature de jeunesse. Point de départ d'une représentation de la petite enfance - Sophie a quatre ans dans Les Malheurs de Sophie - nous considérons que le dialogue ségurien est un apprentissage de soi que met en scène l'auteur pour son personnage. Nous irons plus loin, et supposerons que ce dialogue est aussi, pour l'auteure elle-même confrontée à la nécessité d'inventer, l'expression d'un dialogue interne.

À partir de ce préambule, nous analyserons le dialogue mis en scène dans Les Malheurs de Sophie de façon à la fois particulière et générale. Nous défendrons l'idée que le dialogue, en tant que processus général pour penser, est utilisé par Ségur pour inventer avant de devenir chez elle une manière singulière de raconter.

\section{Le dialogue comme genre littéraire, et la littérature d'enfance et de jeunesse}

En l'un de ses sens, le dialogue est un genre littéraire représentant un échange entre locuteurs. Ce genre fut longtemps associé à l'argumentation, à la rhétorique et à la didactique. La littérature éducative a beaucoup emprunté à cette forme depuis le haut Moyen Âge 4 , reprenant une tradition héritée de Platon, via Augustin. Cette tradition s'est développée au XVIe siècle et elle a continué sous des formes nouvelles aux XVII et XVIII siècles.

\footnotetext{
3. Voici la définition par Vygotski du brouillon mental : «La forme écrite aide au déroulement d'un langage relevant de l'activité complexe. C'est là justement ce qui fonde le recours au brouillon. Le cheminement du "brouillon" au "texte" est précisément une activité complexe. Mais, même si on ne fait pas réellement de brouillon, l'élément de réflexion est très important dans le langage écrit; très souvent nous nous disons d'abord pour nous-mêmes ce que nous allons écrire; il s'agit là d'un brouillon mental. Ce brouillon mental du langage écrit est aussi un langage intérieur. » Lev Semeionovitch Vygostki, La Méthode instrumentale en psychologie, Moscou, 1930/1985, p. 363. Cité par B. Schneuwly et J.-P. Bronckart, Vygotski Aujourd'hui, Lausanne, Delachaux et Niestlé, 1985. 4. Marie-Anne Polo de Beaulieu, Formes dialoguées dans la littérature exemplaire du Moyen Âge, Paris, Champion, 2012.
} 
Dans Le Magasin des Enfants, par exemple, Mme Leprince de Beaumont met en scène une gouvernante et des enfants auxquels sont racontées des anecdotes, des fables, ou des histoires (comme La Belle et la Bête) suscitant une discussion et une morale éducatives, mais distinctes du conte lui-même.

C'est encore le cas des dialogues de théâtre de Mme de Genlis, selon une visée héritière de l'œuvre de Mme de Maintenon.

Isabelle Nières-Chevrel note que :

cet emploi du dialogue pédagogique s'efface progressivement au XIXe siècle. Seul subsiste le récitcadre servant à introduire la parole maternelle dans les recueils d'historiettes, la parole savante dans les ouvrages de vulgarisation scientifique. Tout autre est l'usage du roman discursif tel que nous le trouvons chez Ségur et Collodi 5 .

Et en effet, on ne reconnaît chez Ségur ni les dialogues théâtraux dispensateurs de discussions morales destinées à l'éducation des filles, ni le récit-cadre ouvrant à la parole maternelle décrit plus haut. Il est intéressant, par exemple, de comparer les romans de la trilogie ségurienne avec un recueil que Ségur avait eu en mains dès le début de la rédaction de sa trilogie : Douze Histoires pour les enfants de quatre à huit ans par une mère de famille 6 . Le dialogue théâtralisé y occupe la plus grande place, et Rémi Saudray ${ }^{7}$ a montré de façon très convaincante la parenté thématique d'un dialogue extrait de l'une de ces Histoires («La Poupée de foire») avec un dialogue des Petites Filles Modèles. Cependant les Douze Histoires sont écrites sous la forme de petits dialogues avec moralités, à l'intention édifiante sans ambiguïté, alors que dans Les Malheurs de Sophie, la visée des dialogues, plus complexe, n'est pas univoque.

Bien sûr, au $\mathrm{XX}^{\mathrm{e}}$ siècle et de nos jours, le dialogue théâtralisé pour la jeunesse a pris une forme autonome 8 : le dialogue à usage éducatif sous une forme codifiée n'a plus cours; de nouvelles formes, l'album, la bande dessinée, s'y substituent, parfois sous forme de questionsréponses (voir Robert Kraus 9 , Éric Hill10, auteurs d'albums destinés aux plus jeunes enfants) avec des visées multiples et surtout le concours majeur de l'image11.

Il est probable que la parole, longtemps associée à celle du conteur qui fait autorité, se dissémine au $\mathrm{Xx}^{\mathrm{e}}$ siècle, avec la multiplicité des voix narratives et la variété des formes romanesques : il faut à présent entendre le dialogue au sens privilégié des différentes manifestations

5. Isabelle Nières-Chevrel, «La littérature d'enfance et de jeunesse entre la voix, l'image et l'écrit» [en ligne] sur le site de vox-poetica.com.

6. Ségur demande explicitement à son éditeur Émile Templier, gendre de Hachette, de lui envoyer ce livre, dans la lettre du 29 avril 1858 : «Ayez la bonté de m'envoyer deux ex. des Douze Histoires pour les enfants. Veuillez me dire aussi si Les Malheurs de Sophie que je suis en train de terminer et qui sont des histoires d'enfants très jeunes, seront imprimés avec les caractères des Douze Histoires; je désirerais beaucoup que vous les fassiez imprimer avec ces caractères si faciles à lire pour les enfants. » Voir Comtesse de Ségur, Euvres, «Lettres à son éditeur», édition établie et annotée par Claudine Beaussant, Paris, Robert Laffont et Librairie Hachette, coll. «Bouquins», t. I, 1990, p. LXIX.

7. Rémi Saudray, «À propos des Douze Histoires pour les enfants de quatre à huit ans», Cahiers Séguriens, $\mathrm{n}^{\circ} 9$, Aube, Association des Amis de la Comtesse de Ségur, 2010, p. 126.

8. Pierre Gripari, par exemple, a écrit des pièces de théâtre pour enfants, comme : Pièces enfantines, Paris, Âge d'homme, 1977, et Inspecteur Toutou, Paris, L'école des loisirs, «Neuf en poche», 1986 (rééd.).

9. Robert Kraus, Où vas-tu petite souris?, Paris, L'école des Loisirs, 2004.

10. Éric Hill, Où est Spot mon petit chien?, Paris, Nathan, 1980.

11. Isabelle Nières-Chevrel, art. cit. (note 5). 
linguistiques d'un échange entre locuteurs; sous le vocable «dialogue» se regroupent les représentations de la parole; elles renvoient au discours rapporté ou représenté, notamment dans le roman. La place du dialogue ainsi entendu a grandi dans le genre romanesque, en littérature générale comme en littérature de jeunesse. Un article récent ${ }^{12}$ consacré aux mesures quantitatives des différentes formes d'«oral représenté» dans le genre romanesque a pris pour exemple un classique du $\mathrm{xx}^{\mathrm{e}}$ siècle de la littérature de jeunesse : Les Contes de la rue Broca de Pierre Gripari. Il nous apprend que, comparé à trois autres romans de la littérature classique, le taux de présence des dialogues au discours direct est le plus élevé chez Gripari : $41 \%$, contre $36 \%$ pour un roman de Duras, 32,5\% pour La Chartreuse de Parme.

Et l'article cité en étudie toutes les manifestations, remarquant par exemple que le monologue intérieur existe dans les Contes de Gripari, en proportion cependant moindre (3,4\%) que dans les autres romans classiques anciens et contemporains auxquels il est comparé.

On peut dire que Ségur va découvrir un levier puissant et novateur dans la manière d'inventer en présentant ses personnages par le moyen du dialogue.

\section{Présentation du manuscrit}

Le manuscrit dont nous avons pu disposer grâce à un collectionneur privé, M. François Labadens, est celui que Ségur a communiqué à son éditeur, lequel le lui renvoyait habituellement avec ses remarques au crayon. Il a été relié et conservé par Olga de Pitray, fille benjamine de la Comtesse, à laquelle cette dernière envoyait ses œuvres pour sa collection. Il est peu raturé, certaines pages pas du tout, notamment la première, ce qui semble indiquer que le texte a été recopié et que les premiers états ne nous sont pas parvenus, d'autant que le manuscrit est manifestement dans la phase pré-éditoriale décrite par Pierre-Marc de Biasi ${ }^{13}$. Mais l'examen des premiers chapitres montre pourtant que Ségur a rédigé et modifié des éléments très importants du roman, comme le nom de l'hérö̈ne d'abord appelée Marie, le changement transformant Pauline en Paul, les liens de parenté, notamment les hésitations entre tante ou mère à propos de Madame de Réan, autant même que le nom de Réan 14 , etc., si bien que l'essentiel du texte semble bien avoir été écrit sous l'impulsion de l'invention, avec des ratures rétrospectives palliant les incohérences une fois l'idée trouvée en cours de route (voir le paragraphe suivant). L'écriture, en tout cas, n'est pas celle d'un copiste, mais bien de Ségur, comme le montre la comparaison de l'écriture du manuscrit des Malheurs de Sophie ${ }^{15}$ avec celle de ses lettres ${ }^{16}$. Nous le savons aussi par sa correspondance : Ségur écrit (et réécrit) le manuscrit de sa main et il est possible qu'elle écrive au crayon d'abord

12. Bénédicte Pincemin et Denise Malrieu, «Caractérisation quantitative de textes. Application à l'oral représenté, en diachronie», Textes, documents, œuvres - Perspectives sémiotiques (en hommage à François Rastier), D. Ablali, S. Badir et D. Ducard (dir). Actes du Colloque de Cerisy (6-13 juillet 2012), Rennes, Presses universitaires de Rennes, 2013.

13. Pierre-Marc de Biasi, La Génétique des textes, Paris, Nathan Université, coll. «128», 2000, p. 44 sq.

14. Toutes ces informations ont été notées par les spécialistes (Isabelle Nières-Chevrel, Hortense Dufour, Alain Lanavère, et plus particulièrement par R. Saudray et F. Labadens, art. cit. p. 23 sq.). Le nom de Mme de Réan sera définitif après le chapitre III, où sont visibles les ratures la nommant Mme de Morel, mais le nom de Sophie est fixé dès la fin du chapitre II (après le folio 12 du manuscrit).

15. Pour désigner le manuscrit et ne pas alourdir le texte, nous abrégerons désormais cette référence en Sophie. 16. La Comtesse de Ségur, Correspondance, préface de Michel Tournier, édition établie par Marie-José Strich, Paris, Éditions Scala, 1993, p. 133. 
pour recopier ensuite ${ }^{17}$, ce qui peut aussi expliquer le petit nombre de ratures constatées sur certaines pages du manuscrit dont nous disposions.

Louis Hay 18 avait jadis opposé «écriture à programme» et «écriture à processus » pour signaler deux grands types d'écriture d'auteur. Quelques années plus tard, Pierre-Marc de Biasi 19 renouvelle cette partition en opposant l'«écriture à déclenchement rédactionnel», qui conduit l'auteur à rédiger immédiatement sans se fonder sur un plan écrit, avec des ramifications et modifications nombreuses insérées au fil de la rédaction, et parfois des retours en arrière, et l' «écriture à programmation scénarique», qui conduit l'auteur à préparer la rédaction par des plans ou scénarios parfois très rédigés, à partir d'enquêtes préliminaires, documentation à l'appui.

Ségur appartient clairement à la première catégorie. Elle ne s'appuie manifestement pas sur un plan préétabli. Dans une lettre du 28 janvier 1870, bien postérieure à la Trilogie de Fleurville et adressée à son éditeur Émile Templier (gendre de Hachette), la Comtesse parle ainsi de son dernier manuscrit Après la Pluie le Beau Temps :

Si j'avais pu vous consulter en composant, j'aurais suivi votre plan de la fin, mais vous savez que je n'ai malheureusement pas la faculté de faire d'avance mon canevas ; j'écris au bout de ma plume, sans me douter - en commençant mon livre et même les chapitres - de ce qu'ils contiendront et où ils me mèneront. J'écris à la grâce de Dieu20.

Des lettres antérieures la montraient en effet «sans idée», notamment pour la rédaction de François le Bossu, comme elle l'écrit à sa fille Olga de Pitray le 7 novembre 1863 :

J'ai commencé mon livre Le Petit Bossu. Je n'ai pas d'idée, je n'ai pas de plan, j'écris tout de même, seulement, je crains pour ma réputation 21 .

Quelques jours plus tard, cependant, le 11 novembre 1863, elle écrit, toujours à Olga :

[...] j'en ai cent pages écrites ; les deux cents autres iront plus vite parce que j'ai enfin trouvé une idée, au lieu d'errer dans une lande peuplée de chardons et de joncs marins 22 .

Le 21 novembre 1863, elle annonce à Olga :

J'avance assez rapidement mon Petit Bossu qui arrive à cent quatre-vingt-dix pages et que j'espère terminer ici [à Kermadio]. Élisabeth [une autre de ses filles] le trouve charmant et amusant; mais il a besoin d'être revu et corrigé, car le défaut d'idées et de plan au commencement du livre amène des incohérences qui doivent disparaître...23

17. Elle écrira ainsi à l'un de ses fils, Anatole, le 2 octobre 1867, à propos de ses Évangiles, écrits sous le contrôle de son autre fils, Gaston de Ségur : «J'abrège pour copier vite, vite mes Évangiles, tout est fini : il ne s'agit plus que de copier les 200 premières pages écrites au crayon.» Correspondance, op. cit., p. 161.

18. Louis Hay, «La troisième dimension de la littérature», Texte, $n^{\circ}$ 5/6, 1986-1987, p. 313-328

19. Pierre-Marc de Biasi, La Génétique des textes, op. cit., p. 32 sq.

20. Comtesse de Ségur, Lettres à son éditeur, dans Euvres, C. Beaussant (dir.), Paris, Robert Laffont, coll. «Bouquins», t. I, 1990, p. CXXXVIII.

21. Correspondance, op. cit., p. 131

22. Ibid.

23. Ibid, p. 134. 
Ces extraits confirment que Ségur écrit vite, voire très vite, et au fil de la plume; elle corrige après-coup, une fois l'idée trouvée et l'invention en train, après relecture (d'elle-même ou de ses proches), ce qui peut se passer plusieurs jours (ou semaines) après le début de l'écriture. Elle peut alors envoyer une copie de son manuscrit à l'éditeur et remanier encore, en fonction des remarques de celui-ci. Dans le cas de Sophie, nous savons que l'écriture des Vacances fut entreprise simultanément avec celle de notre manuscrit et qu'elle fut interrompue au bout d'une soixantaine de pages pour défaut d'invention romanesque 24 . Avec Sophie et ses anecdotes brèves et autonomes sans intrigue principale, la rédaction s'effectue aisément (de début mars à fin avril 1858, notent R. Saudray et F. Labadens ${ }^{25}$ ), jusqu'au dernier chapitre : c'est au moment de finir le manuscrit que se serait effectuée la jonction avec l'organisation générale de la trilogie; le développement de l'intrigue des Vacances aurait donné à Ségur l'idée du départ en Amérique des protagonistes de Sophie : ainsi se justifierait le récit ultérieur de Sophie dans Les Vacances, et s'expliquerait le passage de Sophie de Réan à Sophie Fichini des Petites Filles Modèles, sur lequel s'interrogent les petits héros des Vacances ${ }^{26}$.

\section{Dialogue théâtralisé ou discours direct : les hésitations du manuscrit}

Dans Sophie, les dialogues théâtralisés sont les plus visibles et semblent constituer le plus clair du substrat narratif du roman ${ }^{27}$. La correspondance nous apprend qu'ils sont un choix d'écriture. Dans la lettre du 20 février 186128 à son éditeur à propos de «Pauvre Blaise», Ségur indique en post-scriptum :

J'ai aussi oublié de vous demander de conserver les personnages dans le dialogue de l'ouvrage; les noms formant scène donnent beaucoup plus de vie au dialogue : les dit-il, répondit-elle, reprit-il, s'écria-t-il, continua-t-elle, sont fatigants et engourdissent l'action.

Sophie est avant tout un roman. C'est ce qui explique que Ségur hésite souvent entre la forme théâtralisée et le discours direct pour présenter les dialogues entre ses personnages, les deux formes coexistant dans tous les chapitres du roman ${ }^{29}$.

S'agit-il d'un artifice de présentation qui ferait équivaloir dialogue de théâtre et discours direct? Nous ne le pensons pas.

Il faut rappeler que les deux formes ne sont pas substituables : la forme théâtralisée dispense de la présence d'un narrateur rapportant les paroles du personnage au discours direct, pour les présenter directement en indiquant seulement le nom du personnage locuteur (Sophie, la bonne, Mme de Réan ou la maman, etc.). Cette présentation directe est parfois assortie d'une didascalie («en rougissant», «ensemble», «baissant les yeux », etc.), ce que,

\footnotetext{
24. Rémi Saudray et François Labadens, art. cit., p. 23 et $s q$.

25. Ibid., p. 20.

26. Voir aussi Isabelle Nières, «Les Vacances de la Comtesse de Ségur ou Pour en finir avec le malheur de Sophie », La Revue des livres pour enfants, $\mathrm{n}^{\circ} 131-132,1990$, p. 62-73.

27. Tous les autres romans de Ségur sont aussi écrits avec des dialogues théâtralisés.

28. Lettre 35, Paris 20 février 1861, à Émile Templier dans Comtesse de Ségur, Euvres, t. I, op. cit., p. LXXVIII. 29. À l'exception du chapitre VIII «Les sourcils coupés», où l'on ne trouve aucune forme de dialogue théâtralisé, peut-être parce qu'il s'agit d'un chapitre court qui formait initialement un seul chapitre avec le précédent «Les cheveux mouillés », comme le montre le titre raturé du chapitre VII.
} 
d'ailleurs, le narrateur peut généralement faire aussi quand il introduit le discours rapporté sous une forme narrativisée, par exemple, au chapitre XVI («Les fruits confits») :

Sophie et Paul se regardaient d'un air malheureux. «Demande à maman de l'ouvrir», dit tout bas Sophie à Paul. [nous soulignons]

En revanche, le narrateur peut donner son point de vue sur son personnage grâce au verbe introduisant ses paroles ou son attitude («insinua-t-il », «reconnut-il», «crut-il bon de dire», etc.) alors que le dialogue théâtralisé ne le peut pas par définition.

Une autre différence porte sur l'enchâssement des discours rapportés.

Le dialogue thêâtralisé, que Ségur veut animé et reproduisant la vivacité du dialogue qu'il mime, intègre parfois des récits rapportés au discours direct. C'est le cas au chapitre XV «Élisabeth», où le dialogue entre Mme de Réan et Sophie est théâtralisé; mais l'histoire d'Élisabeth et de sa bonne que rapporte Mme de Réan à Sophie ne peut être à son tour théâtralisée : elle est rapportée par la narratrice au discours direct. On comparera avec une scène de conversation au discours direct entre Sophie et la bonne, au chapitre IV ( «Les petits poissons »), dans laquelle celle-ci rapporte à Sophie une scène enchâssant d'autres discours directs. Or, si l'on peut enchâsser un (ou des) discours direct(s) dans un premier discours direct, il est impossible d'enchâsser une réplique théâtralisée dans une autre de même type : les paroles rapportées par un personnage de théâtre sont obligatoirement représentées sous forme de discours direct enchâssé et non comme de nouvelles répliques de théâtre.

On voit donc que les deux formes - la présentation théâtralisée du dialogue et le discours direct rapporté par un narrateur - sont bien deux formes distinctes dont le fonctionnement n'est pas identique.

En dépit de ce qu'elle dit à son éditeur, il est manifeste que Ségur hésite entre ces deux formes. Les ratures nous montrent qu'elle remplace fréquemment l'une par l'autre, en repérant intuitivement leur fonctionnement.

La forme théâtrale est préférée lorsque l'action prend un tournant dramatique ou qu'elle s'infléchit de façon décisive, lorsqu'un conseil ou une interdiction maternels par exemple sont formulés, ou lors d'une dispute (entre Paul et Sophie) ou encore lorsqu'un débat, une justification ou une enquête («Les petits poissons») sont en cours. Les propos théâtralisés en scène sont alors présentés comme le moteur de l'action nouvelle ou de la péripétie : présentés, et non représentés.

Ainsi Ségur remplace-t-elle le discours direct de son premier jet par du dialogue théâtralisé à cinq reprises dans le manuscrit : aux folios 28, 77, 136, 187 et 193 pour présenter une scène spectaculaire ou souligner le rôle d'un personnage.

Dans l'exemple suivant (extrait du chapitre XX, «La petite voiture»), à la faveur d'un ajout en marge qui met en scène André, l'un des deux petits fermiers passagers de la voiture à laquelle est attelé l'âne, on observe une modification de l'intrigue visant à atténuer la responsabilité de Sophie : l'idée d'aiguillonner l'âne avec une branche de houx, initialement de Sophie dans le premier jet, revient finalement à André. La rature, qui remplace une parole de Sophie au discours direct, devient alors un dialogue théâtralisé; mais Ségur oubliera de supprimer le verbe introducteur (« dit Sophie») qui sera conservé jusque dans les éditions contemporaines.

\section{Pourquoi les deux formes coexistent-elles chez Ségur?}




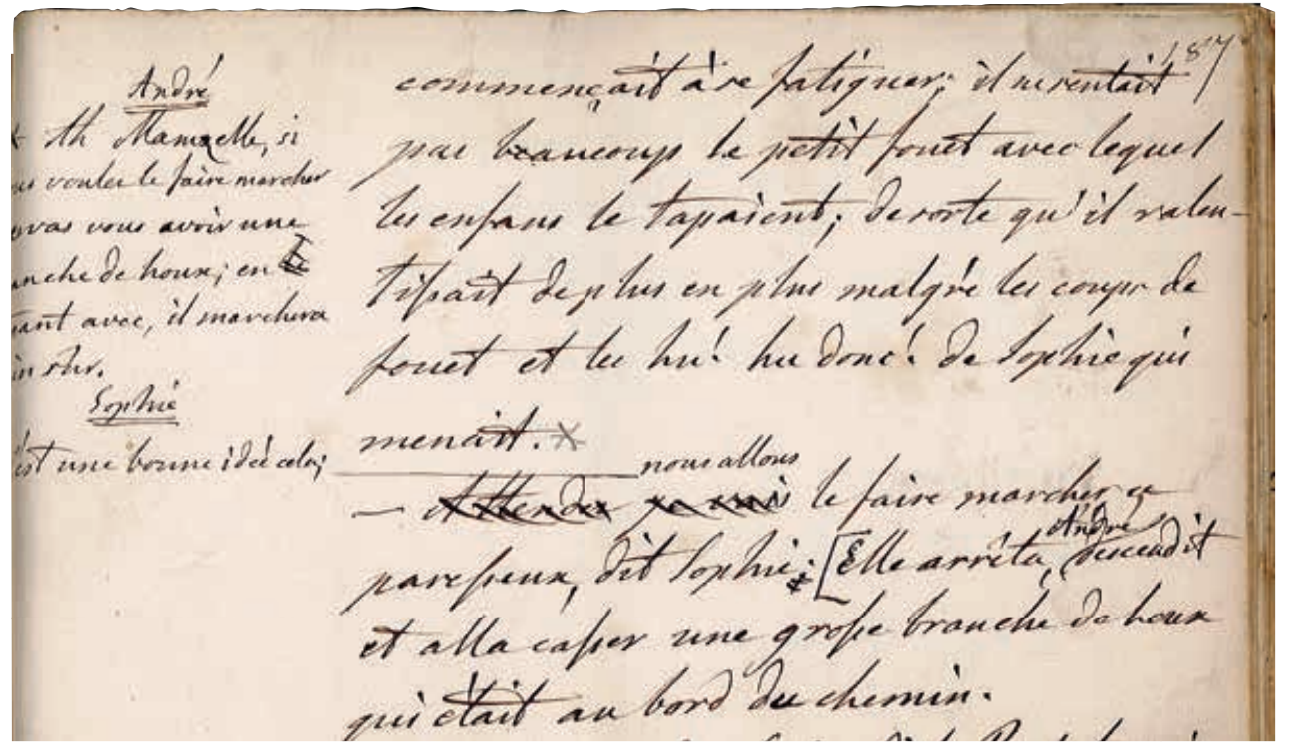

Fig. 1 : Folio 187 , «La petite voiture», chapitre XX. Avec l'aimable autorisation de F. Labadens.

André

Ah! Mamzelle, si vous voulez le faire marcher je vais vous avoir une branche de houx; en le tapant avec, il marchera bien sûr

Sophie

C'est une bonne idée cela; à se fatiguer; il ne sentait pas beaucoup le petit fouet avec lequel les enfants le tapaient; de sorte qu'il ralentissait de plus en plus, malgré les coups de fouet et les hu! hu donc! de Sophie qui menait.*

\section{nous allons}

- Attendez je vais le faire marcher ce

$$
\text { André }
$$

paresseux, dit Sophie; [Elle arrêta, descendit et alla casser une grosse branche de houx qui était au bord du chemin.

Nous trouvons la même présentation au folio 193, lorsque Lambert (qui était nommé Boulard dans le manuscrit, comme dans la réalité vécue de Ségur) vient annoncer la mort de l'âne : Ségur rature «répondit Boulard d'un air triste» et installe une saynète théâtrale permettant à Boulard de faire son récit.

Mais le manuscrit présente aussi beaucoup d'exemples inverses de remplacement d'une forme théâtrale par du discours direct, notamment dans les folios 32, 33, 58, 101, 103, 191 du manuscrit, où la forme narrative avec incise introduit des discours directs. 
Bien qu'il soit difficile de donner une explication absolue de cette alternance, on remarque quelques régularités : ainsi, lorsque le dialogue est caractérisé par une réplique isolée ou par une réponse à un discours direct, celle-ci n'apparaît pas sous forme théâtrale. C'est le cas par exemple au folio 33 où le nom de «Sophie», d'abord placé comme nom de personnage, est raturé et remplacé par l'incise « dit Sophie».

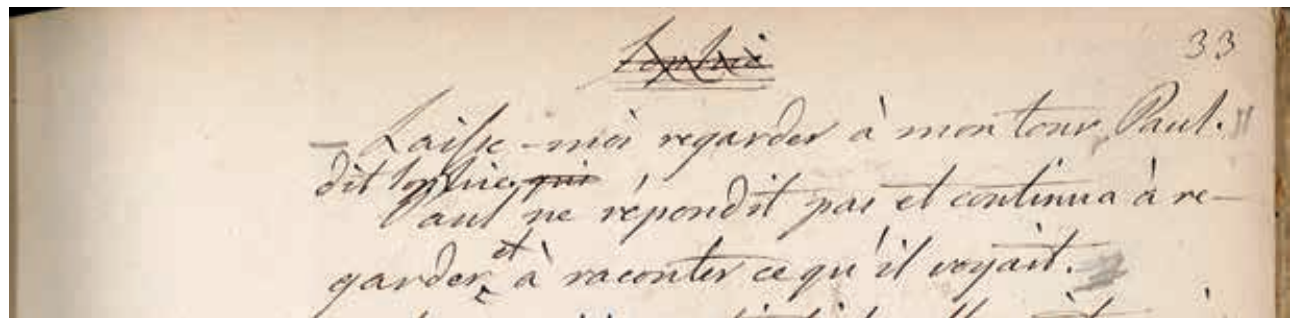

Fig. 2 : Folio 33, «L'abeille», chapitre VI. Avec l'aimable autorisation de F. Labadens.

Sophie
$\begin{aligned} & \text { - Laisse-moi regarder à mon tour, Paul } » \\ & <\text { dit Sophie }>\text { qui } \\ & \text { Paul ne répondit pas et continue à re- } \\ & <\text { et }>\end{aligned}$
garder, à raconter ce qu'il voyait.

On le voit aussi au folio 58, dans lequel l'intitulé «La bonne», présent dans le manuscrit, disparaît de l'édition parce qu'il s'agit d'une réponse à un discours narratif.

Il apparaît ainsi que la frontière entre discours directs rapportés et présentation en dialogues théâtralisés, franchie par Ségur, ne l'est nullement de manière anarchique. Si la représentation du discours direct des personnages s'effectue sous la forme très voyante des dialogues théâtralisés, elle ne remplace en aucune façon les autres formes de représentation des discours directs moins visibles, et tout aussi présentes dans le roman.

Parmi celles-ci, se détachent les discours intérieurs de Sophie.

C'est que le dialogue chez Ségur n'est pas un artifice argumentatif ou rhétorique à visée Les discours intérieurs édifiante : plus que la narration, il construit le roman et le fait avancer.

Les idées qui viennent à Sophie sont généralement exposées par Sophie elle-même, avant leur réalisation, au cours du chapitre dont le titre traduit le contenu à venir. Mais ce que la narratrice appelle les «idées » de Sophie aboutit immanquablement à ses «malheurs ».

30. Notre propos n'est aucunement de traiter de l'endophasie en littérature comme s'y sont brillamment essayés Gilles Philippe (Le Discours en soi. La représentation du discours intérieur dans les romans de Sartre, Paris, Honoré Champion, 1997) et Gabriel Bergounioux (Le Moyen de parler, Paris, Verdier, 2004). Nous voulons seulement suggérer que le choix privilégié de la forme du dialogue dans les romans de Ségur, et spécialement dans Les Malheurs de Sophie, est un moyen pour inventer. 
Cette régularité dans la répétition nous paraît constituer un schème de l'invention chez Ségur : selon notre hypothèse, l'auteure expose, in statu nascendi, les idées de Sophie où sont élaborées les étapes de l'action future sous la forme d'un monologue intérieur, proféré pour le lecteur dans le temps fictif où elles apparaissent. Et c'est le récit ultérieur de leur réalisation malheureuse qui constitue le principe de progression du roman.

Mais la séquence qui va du monologue intérieur à l'enchaînement d'actions du roman peut aussi être dite réflexive, parce que les «idées » données à voir, représentées par le discours intérieur du personnage, constituent pour l'auteure une programmation. On proposera donc l'hypothèse que le dialogue est, chez Ségur, le moteur de l'invention ${ }^{31}$.

Dans Sophie, les monologues intérieurs sont en effet nombreux et se retrouvent dans les deux tiers du roman : seuls sept sur vingt-deux chapitres n'en contiennent pas.

On ne sera pas surpris de constater que du contenu romanesque apparaît au cours d'un monologue intérieur de Sophie montré au lecteur «en train de se faire» et précédant l'action. Il ne faut pas voir de contradiction ici entre monologue intérieur et dialogue : en effet, Sophie se parle à elle-même, faisant apparaître dans un langage intérieur donné à voir au lecteur, hésitations et suppositions. Leur point commun est de présenter les paroles de Sophie comme rapportées par le narrateur au discours direct, annonçant l'action à venir, au lieu de narrer l'action elle-même.

\section{La forme des discours de Sophie}

Cependant, s'agit-il des pensées de Sophie, ou de ses paroles? Ses énoncés sont construits comme des phrases de communication; ce ne sont pas des bribes inachevées qui témoigneraient comme le décrit Vygotski, d'un langage intérieur dont la pensée excède le langage. Ségur présuppose de façon très conventionnelle que l'idée précède l'action, ce qui naturalise les paroles prononcées par Sophie dans la représentation de ses monologues.

Sophie est, en effet, montrée parlant ses idées : comme ses prédécesseur(e)s des XVII et XVIII siècles, Ségur emploie plutôt «dire» que «se dire», verbum dicendi plutôt que verbum sentiendi : sur les vingt-cinq occurrences de monologues intérieurs que compte le manuscrit, c'est le verbe «dire» qui prédomine, accompagné d' «ajouter» et de «s'écrier», alors que Sophie est seule. Et cinq de ces occurrences seulement comportent le «se» réflexif (folios 4, 14, 15, 20, 78) même si l'on note que Ségur l'ajoute après relecture pour deux occurrences (folios 9, 36), ce qui les porte à sept sur vingt-cinq. À l'inverse, «se» est barré à deux reprises dans les folios 30-31 et 119.

Il arrive que Ségur emploie aussi le verbe «penser» (folios 100, 118, 119, 166-167), mais cet emploi arrive tardivement dans le manuscrit, et reste marginal.

La forme parlée utilisée par Sophie pour «dire ses pensées» ressemble beaucoup à ce que Piaget a nommé le «langage égocentrique de l'enfant» mais dont Vygotski s'est appliqué à démontrer la complexité : car Sophie parle ce que Vygotski appellera plutôt «une forme transitoire du langage extériorisé au langage intérieur ${ }^{32}$ », nous y reviendrons en conclusion.

\footnotetext{
31. Sur cette question, l'auteur de cet article se permet de citer son ouvrage : Catherine Boré, Modalités de la fiction dans l'écriture scolaire, Paris, L'Harmattan, coll. «Savoir et Formation», 2010, en particulier le chapitre IV. 32. Lev S. Vygotski, Pensée et Langage, traduction de Françoise Sève [1997], Paris, La Dispute, 1934, p. 106
} 


\section{Les «idées» de Sophie (et de Ségur?) ${ }^{33}$}

Nous dirons donc que Sophie est montrée en train de penser à haute voix. Elle réfléchit et Ségur le dit. Chaque fois, l'épisode réflexif est systématiquement accompagné de la présentation du mot métalinguistique «idée», qui devient progressivement un sujet en soi dans le roman.

À partir du folio 80, ce mot d'«idée» va même devenir, par un glissement sémantique, un équivalent de «sottise» ou «bêtise».

Et dans le dernier tiers du manuscrit, les monologues à haute voix cessent : à ce moment-là, c'est au cours de dialogues, avec Paul et parfois avec sa mère, que le personnage de Sophie va exposer ses «idées », qui seront alors ouvertement qualifiées par Mme de Réan de : «qui n'ont pas le sens commun» (voir la rature sur «absurdes» au folio 191, fig. 3).

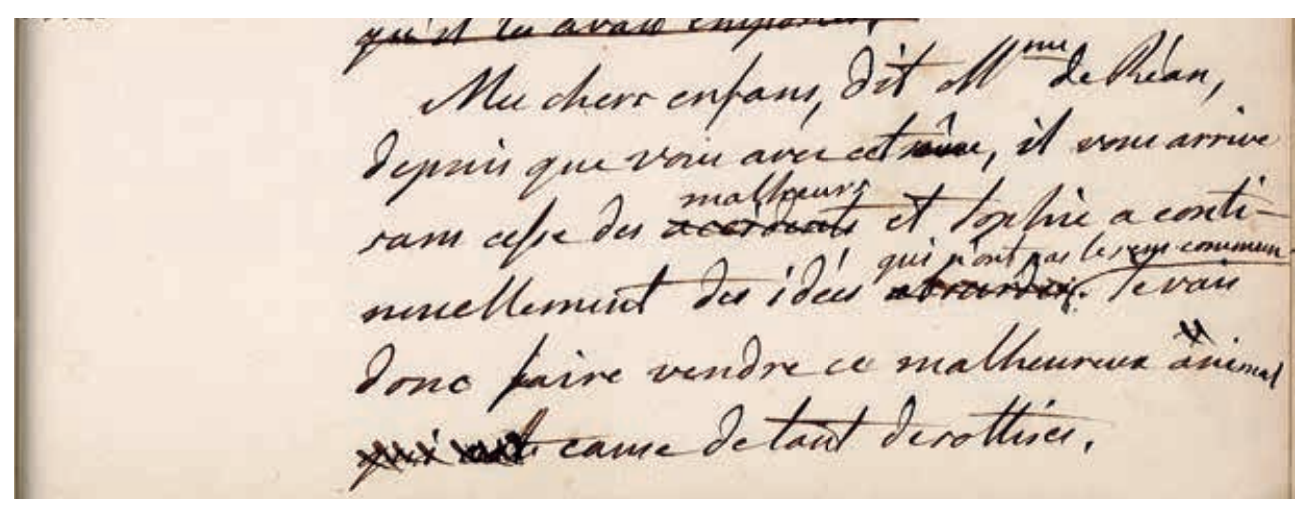

Fig. 3 : Folio 191, «La petite voiture», chapitre XX. Avec l'aimable autorisation de F. Labadens.

Mes chers enfans, dit Mme de Réan, depuis que vous avez cet âne, il vous arrive $<$ malheurs $>$

sans cesse des accidents et Sophie a conti$<$ qui n'ont pas le sens commun $>$ nuellement des idées absurdes. Je vais donc faire vendre ce malheureux ânimal qui est cause de tant de sottises.

33. En dépit d'une assimilation rétrospective, évidente pour le lecteur, entre le personnage de Sophie et le propre prénom de la Comtesse, il est délicat de présenter l'assimilation de l'une à l'autre comme un processus direct et naturel : c'est probablement un mouvement très peu conscient et progressif de la part de Ségur, qui, nous le rappelons, avait d'abord prénommé son hérö̈ne Marie. 
Et dans le folio suivant (192, fig. 4), le mot «idée » a définitivement le sens de «bêtise».

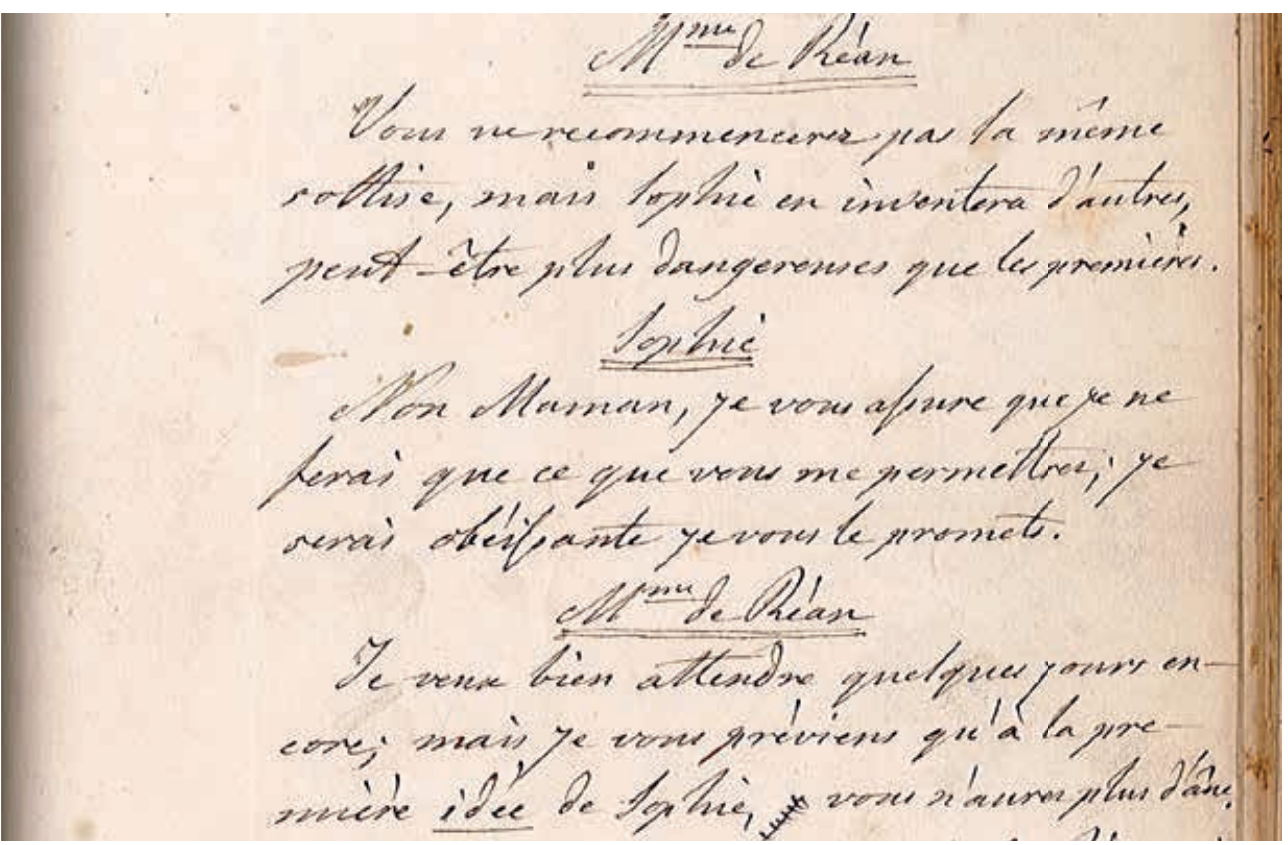

Fig. 4 : Folio 192, «La petite voiture».

Avec l'aimable autorisation de F. Labadens.

\section{$M^{\text {me de Réan }}$}

Vous ne recommencerez pas la même sottise, mais Sophie en inventera d'autres, peut-être plus dangereuses que les premières.

\section{Sophie}

Non Maman, je vous assure que je ne ferai que ce que vous me permettrez; je serai obéissante je vous le promets.

\section{$\underline{M^{m e} \text { de Réan }}$}

Je veux bien attendre quelques jours encore; mais je vous préviens qu'à la première idée de Sophie + vous n'aurez plus d'âne

On peut penser que le dialogue intérieur de Sophie a pu être, au début du roman, un moyen pour Ségur de nourrir l'invention fictionnelle, avant de se transformer en un schéma répétitif : les idées de Sophie engendrent la fiction, identifiée à ses «malheurs » (cf. fig. 4, folio 191, ligne 3). 


\section{Le fonctionnement des discours de Sophie}

Sophie extériorise un discours organisé et surtout prédictif. En effet, ses réflexions sont au futur simple ou périphrastique, car ils annoncent un contenu à venir. On le voit clairement dans les folios 19, 36, 119.

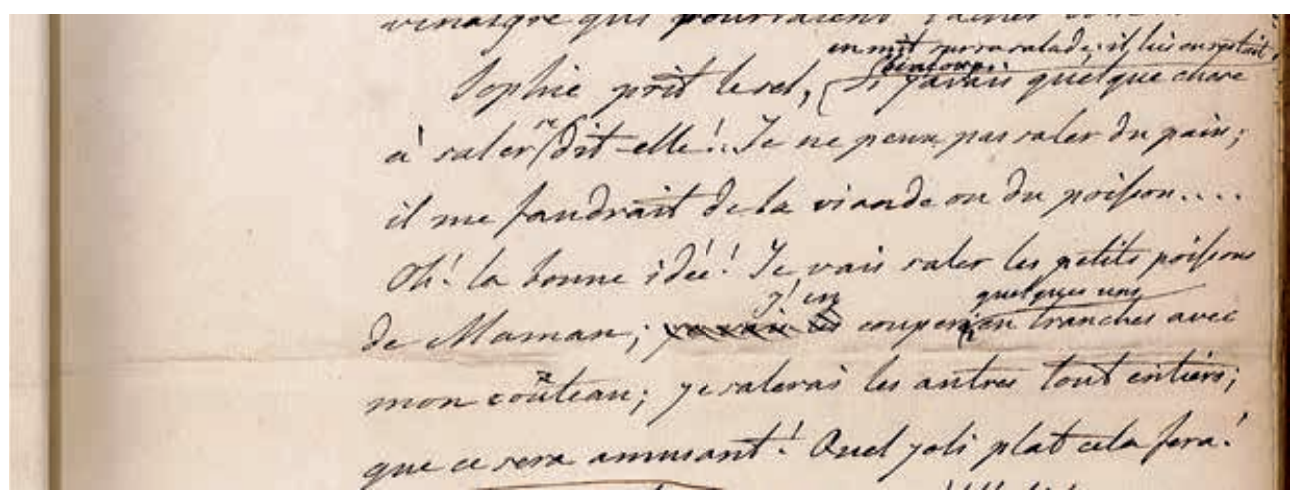

Fig. 5 : Folio 19, «Les petits poissons », chapitre IV. Avec l'aimable autorisation de F. Labadens.

$<$ en mit sur sa salade; il lui en restait. $>$

$<$ encore beaucoup $>$ [en surcharge]

Sophie prit le sel- $<,>$ Si j'avais quelque chose

à saler $<$ se $>$ dit-elle !....Je ne peux pas saler du pain;

il me faudrait de la viande ou du poisson....

Oh! la bonne idée! Je vais saler les petits poissons

$$
\text { j'en } \quad<\text { quelques-uns }>
$$

de Maman; je vais les couper $<$ ai $>$ en tranches avec mon côtteau; je salerai les autres tout entiers; que ce sera amusant! Quel joli plat cela fera!

Au folio 36, «L'abeille», chapitre VI, le manuscrit donne :

Je vais lui couper la tête pour la punir de toutes les piqûres qu'elle a faites,

tandis qu'au folio 119, «Les fruits confits», chapitre XVI, Ségur nous montre Sophie dans l'action, interrompue par sa réflexion :

Sophie elle ne sait comment

faire, elle cherche un bâton, une pincette,

n'importe quoi $<$, > pour atteindre la boîte,

lorsqu'elle se tape le front avec la main

en se disant : $<->$ [Que je suis donc bête $;<$ ! > Je vais

approcher un fauteuil et monter dessus.

Certains extraits reposent parfois sur un système hypothétique exprimant un contenu délibératif : Sophie envisage plusieurs hypothèses avant de trouver l'idée qui l'emportera : 
elle pèse les conséquences et prend sa décision au terme de sa discussion avec elle-même, que ce soit pour transgresser une interdiction, ou pour dissimuler une catastrophe survenue après la réalisation de son «idée» (folios 41, 148-149). Au folio 41, «Les cheveux mouillés », chapitre VII, Sophie réfléchit ainsi :

$-[\mathrm{Si}$

je mouillais les miens dit-elle, ils aient [en surcharge]

friserent peut-être.

Le chapitre XVIII «La boîte à ouvrage » offre notamment un exemple très étendu de délibération interne utilisant un système hypothétique, qui marque un véritable dialogue intérieur chez Sophie.
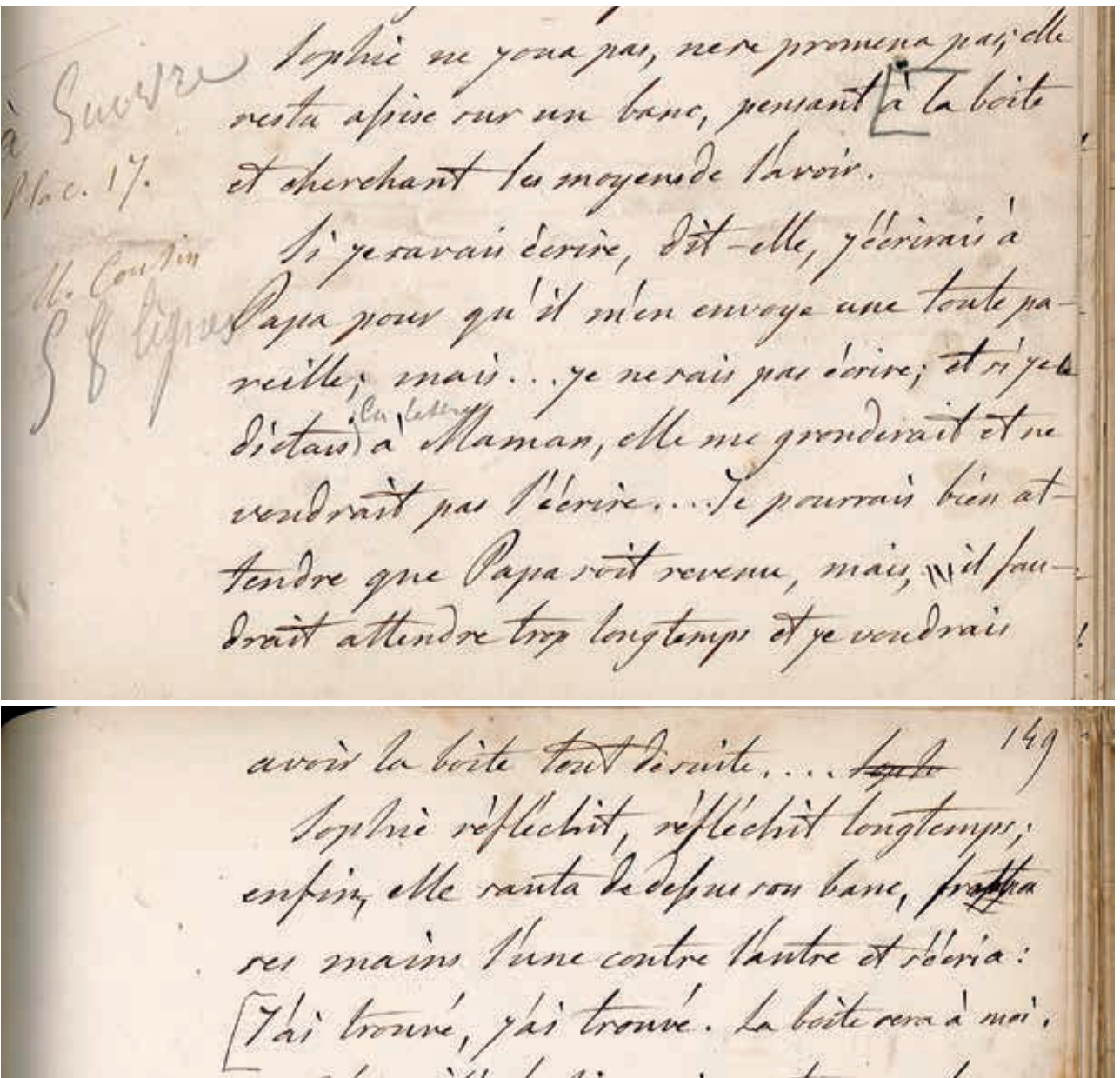

Fig. 6 : Folios 148-149, «La boîte à ouvrage», chapitre XVIII. Avec l'aimable autorisation de F. Labadens. 
Sophie ne joua pas, ne se promena pas; elle resta assise sur un banc, pensant [à la boîte et cherchant les moyens de l'avoir.

Si je savais écrire, dit-elle, j'écrirais à

Papa pour qu'il m'en envoye une toute pareille; mais... je ne sais pas écrire; et si je ta $<$ la lettre $>$

dictais à Maman, elle me gronderait et ne voudrait pas l'écrire....Je pourrais bien attendre que Papa soit revenu, mais,... il faudrait attendre trop longtemps et je voudrais avoir la boîte tout de suite....Soph Sophie réfléchit, réfléchit longtemps;

enfin, elle sauta de dessus son banc, frappa frotta [en surcharge] ses mains l'une contre l'autre et s'écria :

[J'ai trouvé, j'ai trouvé. La boîte sera à moi

Cependant, Ségur nous montre Sophie exposant aussi ses idées par le dialogue avec autrui et c'est dans le dernier tiers du roman que l'on trouve tous les dialogues avec Paul où elle explique ses «idées» (folios 158, 173, 200).

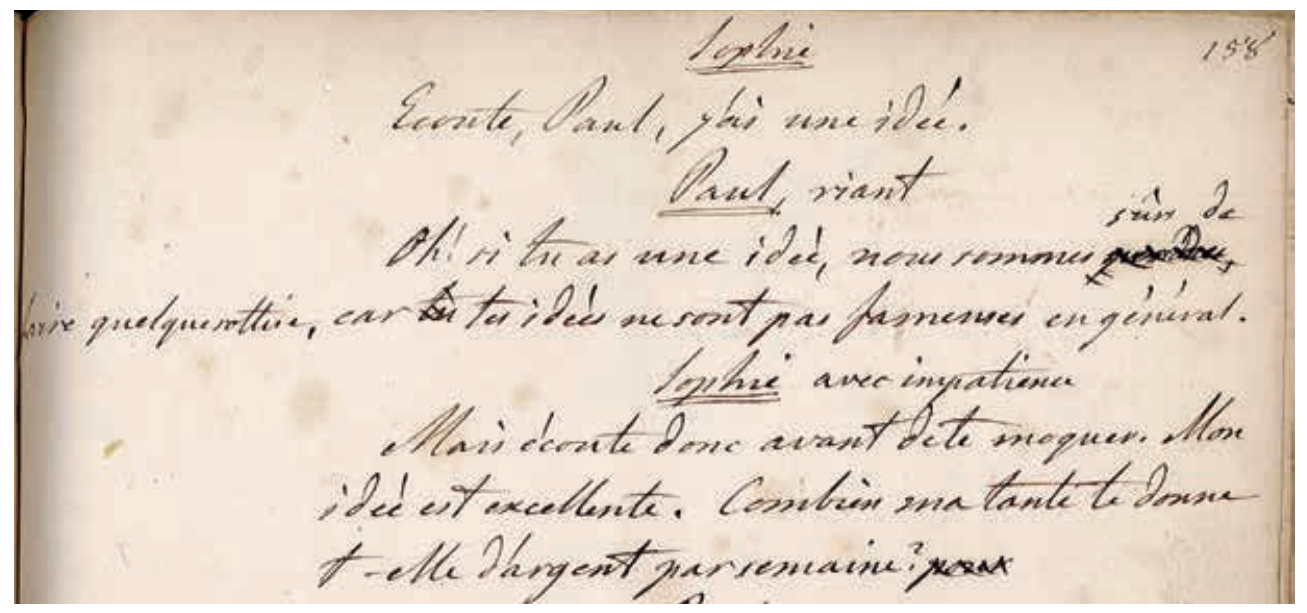

Fig. 7 : Folio 158, «L'Âne», chapitre XIX.

Avec l'aimable autorisation de F. Labadens.

\section{Sophie}

Écoute, Paul, j'ai une idée.

\section{Paul, riant}

$$
<\text { sûrs de }>
$$

Oh! si tu as une idée, nous sommes perdus faire $<$ quelque sottise $>$, car tu tes idées ne sont pas fameuses en général.

Sophie avec impatience

Mais écoute donc avant de te moquer. Mon idée est excellente. Combien ma tante te donne t-elle d'argent par semaine? pour 
La suite de cette scène est une extraordinaire argumentation au cours de laquelle Sophie réfléchit à haute voix et finit par obtenir l'assentiment de sa mère pour l'âne et la petite voiture. Bien sûr, les «idées» suivantes - et Ségur prend la peine de souligner elle-même le mot «idée» dans le manuscrit - quoique discutées avec Paul, entraîneront d'autres malheurs : mort de l'âne, puis deux chapitres plus loin, de la tortue.

Il y a donc eu chez Ségur une compréhension progressive dans la conception du contenu de son roman. La trame - peut-être accidentelle au début - de ce que pense la petite fille «colère, gourmande, menteuse et voleuse» comme la décrit Ségur dans sa préface rétrospective au roman, devient systématique, et le sujet central du roman tournera autour de ce mot «idée », dont l'exposé du contenu est donné à voir dans les dialogues, et qui devient la matrice du roman.

\section{Dialogue et invention}

Avant de conclure, nous reprendrons notre réflexion initiale sur le rapport entre le dialogue et l'invention, si bien thématisé par les «idées» de Sophie.

L'observation de dialogues entre enfants placés dans des situations d'écriture collaborative organisées dans le cadre scolaire permet d'apporter quelques précisions sur la fonction du dialogue dans la création de textes fictionnels, comme le montre ce bref extrait d'un dialogue transcrit entre deux petites filles de 7 ans environ, qui doivent inventer une histoire sur le thème «Comment sont apparus les premiers hommes et les premières femmes » 34 .

411. Laurence [relisant ce qu'elle a écrit] : Le premier eut...

412. Elizabeth [corrigeant] : Et le premier...

413. Laurence [montrant le passage sur la feuille] : Et le premier eut...

414. Elizabeth [dictant] : ... une idée.

[...]

417. Laurence [écrivant] : ... idée.

418. Elizabeth : Non. J'ai une idée. [Relisant le texte :] Il y a un s à la fin de eut.

419. Laurence [écrivant] : Ah, oui, t'as raison... eut une idée. Il dit...

420. Elizabeth [dictant] : «Ah, je vais me transformer en femme.»

421. Laurence : Voilà. «Je vais me déguiser en femme.» C'est mieux.

[...]

440. Elizabeth : «Je vais me transformer», ça veut dire qu'elle est vraiment femme.

L'extrait du texte des deux fillettes résultant de leur travail d'invention porte ci-dessous des traces de ratures au stylo bleu.

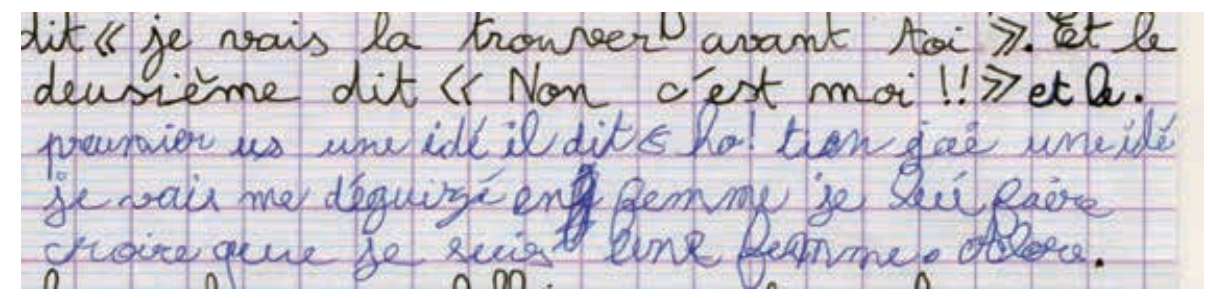

Fig. 8 : Extrait d'écriture collaborative de deux fillettes de sept ans (école élémentaire Paul-Cézanne, Val d'Oise).

34. Dialogue enregistré et filmé en juin 2012, à l'école élémentaire Paul-Cézanne, à Marines dans le Val d'Oise. Les prénoms ont été changés. Comme il s'agit d'une transcription d'un oral où les sujets relisent à haute voix un texte écrit, nous avons choisi de ne pas faire de transcription diplomatique de cet écrit mais de le considérer comme oralisé. 
On retrouve ici l'enfance de l'idée, émergeant du dialogue. Il est frappant de voir que, comme Sophie (et Ségur?), les deux fillettes s'interrogent à haute voix sur les idées qu'elles ont pour faire avancer leur récit. Au milieu du texte que l'une dicte à l'autre, Elizabeth s'interrompt ainsi : «Non. J'ai une idée.» Le dialogue entre enfants interfère avec le personnage qu'elles sont en train de créer dans la fiction écrite («et le preumier us une idée, il dit "ho tien jai une idé" $[\ldots] »)$, ce qui permet une réflexivité récursive.

Pour tout usager de l'école observant les pratiques d'écriture collaborative, ce processus est courant. Il montre une labilité des frontières entre le produit de l'invention (le texte écrit) et le processus métalinguistique favorisé par le dialogue qui lui donne naissance : autrement dit, la réflexion métalinguistique née du dialogue extériorisé entre pairs nourrit l'invention et se nourrit d'elle en retour.

Nous avons, dès l'introduction, signalé notre attachement aux thèses de Vygotski sur la primauté du dialogue comme conduite sociale première pratiquée par le jeune enfant. Dans l'analyse que Vygostki fait de sa différence avec Piaget, au chapitre II de son ouvrage Pensée et Langage, il expose très clairement le mécanisme qui conduit l'enfant, de la discussion ou de la dispute, à la réflexion extériorisée (le langage égocentrique) puis à la pensée intériorisée.

Il [Piaget] a montré comment cette réflexion survient après l'apparition de la discussion véritable au sein d'une collectivité d'enfants, comment c'est seulement dans la dispute, dans la discussion qu'émergent les éléments fonctionnels qui déclenchent le fonctionnement de la réflexion.

Il se produit quelque chose d'analogue, croyons-nous, lorsque l'enfant se met à parler à lui-même exactement comme il le faisait auparavant avec les autres, lorsqu'il commence, tout en se parlant, à penser à haute voix là où la situation l'impose.

Le langage égocentrique, qui s'est détaché du langage social, se transforme ensuite en langage intérieur, base de la pensée tant autistique que logique de l'enfant ${ }^{35}$.

Nous voulons pour finir formuler l'hypothèse qu'un tel mécanisme est à l'origine de toute création, chez l'enfant comme chez l'adulte : de même que, lors du dialogue collaboratif en classe naît une idée commune acceptable par les deux locuteurs, et qui peut dès lors s'élaborer sous une forme distanciée par l'écrit, et même s'y représenter "en train de se faire », de même il nous semble que l'écrivain, en représentant des dialogues, extériorisés entre personnages ou internes à ceux-ci, effectue une projection de ses pensées, affects, ou idées, qui produit un travail de réflexion analogue : ce qu'il écrit modifie sa pensée, entraînant ce que nous pourrions appeler le processus créateur.

Cette intuition du dialogue, moteur de l'invention, nous semble avoir été perçue par un auteur très près de l'enfance, qui s'est appuyé sur la parole de l'enfant en elle, pour la représenter sous les deux formes du dialogue externe entre personnages et du discours intérieur, retrouvant ainsi un processus de création universel. 
CATHERINe Boré est professeure émérite à l'université de Cergy-Pontoise-ESPE. Ses recherches portent sur les processus d'écriture, les styles et les genres observables dans les brouillons scolaires écrits, et plus généralement sur les processus de création issus du dialogisme oral et écrit. Ses travaux s'appuient méthodologiquement sur la génétique des textes, la linguistique de l'énonciation en relation avec la psychologie du développement et la sémiologie de l'éducation. Elle est auteure de Modalités de la fiction dans l'écriture scolaire, L'Harmattan, collection «Savoir et Formation», préface de Frédéric François, 2010.

catherine.lamothe.bore@gmail.com

\section{La Comtesse de Ségur ou l'universel du dialogue}

À partir de l'exemple du manuscrit de l'un des auteurs de jeunesse les plus emblématiques, Les Malheurs de Sophie de la Comtesse de Ségur, l'auteur de l'article s'interroge sur le caractère anthropologique du dialogue dans l'invention. Après en avoir rappelé l'origine à la fois didactique, argumentative et éducative dans la littérature générale et d'enfance, l'auteur analyse ses formes dans le texte du manuscrit. Outre le dialogue théâtralisé dont la fonction est exemplarisante, ce sont les discours rapportés, principalement les monologues intérieurs qui sont analysés. Ils montrent comment Ségur s'en sert réflexivement pour donner sens et finalité au roman.

This paper deals with the famous manuscript Les Malheurs de Sophie by the Comtesse de Ségur, and beyond this, with an anthropological question about what key role dialogue should play in youth literature and fictional creation. This paper first recalls the meaning of «dialogue » as a historical genre, and then studies the main forms of dialogue in the text of the manuscript. In addition to the theatrical dialogue, the author studies direct speech, particularly internal monologues. They show what general meaning Ségur gives to literary representations of childhood by using dialogue in a reflexive manner.

Der vorliegende Artikel behandelt das Manuskript Les Malheurs de Sophie der Comtesse de Ségur, einer der bedeutendsten Jugendbuch-Autorinnen. Anhand dieses Beispiels wird der anthropologische Charakter der Dialoge in Jugendliteratur und Belletristik untersucht. Wir betrachten zuerst den didaktischen, argumentativen und erzieherischen Ursprung des Dialogs in der Literatur im Allgemeinen und in der Jugendliteratur im Speziellen und analysieren auch dessen Formen im Falle der konkreten Erzählung. Zusätzlich zum theatralischen Dialog, dessen Funktion exemplarisch ist, geht es um indirekte Rede und inneren Monolog. An diesen Formen wird gezeigt, wie Ségur sich ihrer bedient, um dem Roman Sinn und Zweck zu verleihen.
A partir del caso del manuscrito de una de las autoras más emblemáticas de la literatura infantil, Las desventuras de Sophie de la Condesa de Ségur, el autor de este artículo se interroga acerca del carácter antropológico del diálogo en la invención. Después de recordar su origen, a la vez didáctico, argumentativo y educativo, en la literatura general y en la infantil, el autor analiza sus formas en el texto del manuscrito. Además de los diálogos teatralizados, cuya función es ejemplificadora, se analizan los discursos indirectos y más particularmente, los monólogos interiores. Estos muestran como Ségur utiliza los diálogos de manera reflexiva para otorgar un sentido y una finalidad a la novela.

A partir do manuscrito de uma autora emblemática, Les Malheurs de Sophie da Condessa de Ségur, este artigo interroga-se sobre o caráter antropológico do diálogo na criação literária. Recordando a origem didática, argumentativa e educativa do diálogo na literatura geral e infantil, são analisadas as formas de que se reveste no manuscrito: por um lado o diálogo teatralizado, de função exemplar, por outro os discursos relatados, especialmente monólogos interiores, que mostram como Ségur os usa em modo reflexivo para dar sentido e propósito ao seu romance.

Partendo dal manoscritto di Les Malheurs de Sophie della Contessa di Ségur, tra gli scrittori per l'infanzia più emblematici, l'autore dell'articolo si interroga sul carattere antropologico del dialogo nell'invenzione. Dopo averne richiamato l'origine a un tempo didattica, argomentativa ed educativa sia nella letteratura generale che in quella per l'infanzia, si analizzano le forme del dialogo nel testo del manoscritto. Oltre al dialogo teatralizzato, la cui funzione è esemplificativa, vengono studiati i discorsi diretti, e in special modo i monologhi interiori; l'utilizzo riflessivo di questi ultimi serve a Ségur per dare senso e finalità al romanzo. 